This is the author's final, peer-reviewed manuscript as accepted for publication. The publisher-formatted version may be available through the publisher's web site or your institution's library.

\title{
On monitoring of multiple non-linear profiles
}

Shih-Hsiung Chou, Shing I. Chang \& Tzong-Ru Tsai

\section{How to cite this manuscript}

If you make reference to this version of the manuscript, use the following information:

Tsai, T. R., Chang, S. I., \& Chou, S. H. (2014). On monitoring of multiple non-linear profiles.

\section{Published Version Information}

Citation: Tsai, T. R., Chang, S. I., \& Chou, S. H. (2014). On monitoring of multiple nonlinear profiles. International Journal of Production Research, 52(11), 3209-3224.

Digital Object Identifier (DOI): 10.1080/00207543.2013.867088

Publisher's Link:

http://www.tandfonline.com/doi/full/10.1080/00207543.2013.867088\#.VDVDSfnF8k0

This item was retrieved from the K-State Research Exchange (K-REx), the institutional repository of Kansas State University. K-REx is available at http://krex.ksu.edu 


\title{
On Monitoring of Multiple Non-linear Profiles
}

\author{
Shih-Hsiung Chou* and Shing I Chang
}

Quality Engineering Laboratory, Department of Industrial and Manufacturing Systems Engineering, Kansas State University, Manhattan, Kansas, USA.

\section{Tzong-Ru Tsai}

Department of Statistics, Tamkang University, Tamsui District, New Taipei City, Taiwan.

*Corresponding Author. Email: cls3415@ksu.edu

Shih-Hsiung Chou is a Ph.D. candidate in the Department of Industrial and Manufacturing Systems Engineering at Kansas State University. His main research interests include multivariate statistical process control, high dimensional data visualization, data mining, and machine learning.

Dr. Shing I. Chang is an associate professor in the Department of Industrial and Manufacturing Systems Engineering at Kansas State University. His main research interests include multivariate statistical process control for manufacturing and health care, nonlinear profile monitoring, neural networks and fuzzy set applications in quality engineering, and multivariate experimental designs. He is a senior member of both Institute of Industrial Engineers and American Associate of Quality. He served as department editor of IIE Transactions in 2003 to 2009. He was a NASA summer fellow in 2004 and a recipient of SME young manufacturing engineer award in 1997.

Dr. Tzong-Ru Tsai is a professor in the Department of Statistics at Tamkang University. His main research interests include quality control and reliability analysis. He has served as an executive editor for the International Journal of Intelligent Technologies and Applied Statistics since 2008 and served in the editorial board of the Journal of the Chinese Institute of Industrial Engineers from 2007 to 2011. Dr. Tsai also served as a senior consultant for the Electronics and Optoelectronics Research Lab. of the Industrial Technology Research Institute in 2010 to 2011. 


\title{
On Monitoring of Multiple Non-linear Profiles
}

\begin{abstract}
Most state-of-the-art profile monitoring methods involve studies of one profile. However, a process may contain several sensors or probes that generate multiple profiles over time. Quality characteristics presented in multiple profiles may be related multiple aspects of product or process quality. Existing charting methods for simultaneous monitoring of each multiple profile may result in higher false alarm rates. Or worse, they cannot correctly detect potential profile relationship changes. In this study, we propose two approaches to detect process shifts in multiple nonlinear profiles. A simulation study was conducted to evaluate the performance of the proposed approaches in terms of average run length under different process shift scenarios. Pros and cons of the proposed methods are discussed. A guideline for choosing the proposed methods is introduced. In addition, a hybrid method combining the salient points of both approaches is explored. Finally, a real-world data set from a vulcanization process is used to demonstrate the implementation of the proposed methods.
\end{abstract}

Keywords: Big Data, Profile Analysis, Multiple Profiles Analysis, Multivariate SPC, Multivariate EWMA.

\section{Introduction}

Quality characteristics represented as profiles have been studied in recent quality monitoring literature in recent years. For examples, Jin and Shi (1999) examined the stamping tonnage data over time within a cycle. Kang and Albin (2000) introduced a calibration issue during the etch step of a semiconductor manufacturing process. Walker and Wright (2002) studied the density of wood board over a section. Chang and Gan (2006) showed the monitoring stability of a calibration process in order to assure its accuracy. Paynabar and Jin (2011) presented pressing force profile signals in a valve seat assembly operation. Chang et al. (2012) investigated the temperature profile from a curing process for high-pressure hose products. These studies only consider one profile type in their respective applications.

Taking the advantage of information technology, engineers no longer measure the quality characteristics by hands but through automatic data sensors. This paradigm shift has resulted in a tremendous amount of data. For example, Jin and Shi (1999) reported that one tonnage sensor within a sampling interval could collect 1500 data points for each part, and the database would store $2.88 \times 10^{6}$ data points for 30 presses in 16 hours of production. Although modern database management systems can handle and store those huge datasets, it is very difficult for conventional multivariate statistical process control techniques, such as, Multivariate Hotelling's $\mathrm{T}^{2}$ control chart, to deal with big data and multiple types of profiles simultaneously.

Profile analysis can be simply characterized into two categories, linear and nonlinear profiles according to the shape complexity of a profile of interest. With respect to linear profile applications, model parameters are the subjects of monitoring because linear profiles are easy to be presented by, for example, a simple linear 
regression model. Many studies monitored either intercept or slope parameter of the calculated simple linear regression model or monitoring both. For example, Kang and Albin (2000) proposed two approaches, the first one monitored slope and intercept with the Hotelling's $T^{2}$ control chart, while the second one monitored average residuals between sample profiles and reference profile followed by exponentially weighted moving average (EWMA) chart and $R$ chart. Kim et al. (2003) showed their method of three univariate EWMA charts monitoring slope, intercept, and the variance of deviation between samples and regression line performs better than EWMA/ $R$ chart in terms of average run length (ARL).

Studies in nonlinear profile analysis can be categorized into four typesapplying multiple and polynomial regression (Zou et al., 2007; Kazemzadeh et al. 2008; Mahmoud 2008), applying nonlinear regression models (Ding et al., 2006; Williams et al., 2007; Shiau et al., 2009; Chang and Yadama 2010; Chen and Nembhard 2011;), use of mixed models (Jensen et al., 2008; Jensen and Birch, 2009; Qiu et al., 2010; Paynabar and Jin, 2011 ), and use of wavelets (Reis and Saraiva, 2006; Zhou et al., 2007; Chicken et al., 2009). For more detail of those methods to monitor the process stability can be found in Woodall (2007) and Noorossana et al. (2011).

The studies mentioned above only consider one profile type for process monitoring. However, data points that collected in a process or system may be characterized by two or more profiles. Noorossana et al. (2010) investigated a calibration application between desired force and the real force produced by 1600-ton hydraulic press machine. The machine consists of a set of cylinders, pistons and hydraulic pipe controlled by a programmable logic controller (PLC) for input and output factors adjustment. The input variable known as the desired force or nominal force is given by a motor placed on the top of machine so that four real forces or the response variables collected from four cylinders of the press can be measured by a PLC. Since four response variables can be considered as correlated linear profiles, Noorossana et al. (2010) proposed a multivariate simple linear profile method to deal with this problem. Specifically, all linear profiles are of the same type but for each press cylinder. Their method cannot monitor multiple correlated nonlinear profiles of different types that measure different process characteristics.

An example of multiple correlated nonlinear profiles can be found in a curing process of high-pressure hose products. According to Chang et al.'s (2012) study, the high-pressure hose products are covered layers of rubber and metal wires, which are loaded and cured in a heated chamber called an autoclave or vulcanizer, equipped with several sensors in different locations for monitoring air temperature, condensation water temperature, and chamber pressure. Although the key factor of curing process is the air temperature, the other profiles, such as, chamber pressure profiles, monitored simultaneously also play important roles in the curing process. Note that, high chamber pressure will increase the speed of reaching target air temperature. Also, a sealed chamber helps air temperature climb quickly and stably to the setup temperature point. On the other hand, a leaking vulcanizer requires more energy consumption to maintain the same temperature during the curing stage. Therefore, it is easier for quality 
engineers to monitor the pressure profile using statistical process control (SPC) tool for saving energy.

Chang et al. (2012) only investigated the air temperature profile of the curing process. When this process is out of control, it is very difficult for quality engineers to pinpoint the root cause. It is possible that the chamber is not airtight but both temperature and pressure maintain their target values. However, the relationship between the temperature and pressure profiles may have changed. In this study, both air temperature and pressure profiles are considered simultaneously. This example provides an illustration of how the proposed framework addresses multiple profile process monitoring in general. This paradigm motivates us to develop a novel approach for simultaneous monitoring of multiple correlated nonlinear profiles. A two-profile simulation study is conducted to evaluate the performance of the proposed charting methods.

Figure 1 shows overall air temperature and pressure profiles that generated from a typical curing process. It may be possible to construct an underlining physics equation between the air temperature and pressure so that the quality characteristics can then be transformed from profiles to parameters as variables used in multivariate control chart. However, this underlining equation is not easy to be formulated, and it cannot be generalized for all applications. In other words, this equation (if it can be formulated) can only be used in this curing process application. In addition, according to Figure 1, it is obvious that the temperature and pressure profile are correlated, and yet, it is hard to define such a correlation between profiles in general. Although monitoring those profile types using multiple multivariate control charts independently provides a solution, high false alarm rate and low detecting power is the major concern given that profiles are correlated to each other. This study provides a general SPC framework for multiple correlated linear or nonlinear profiles.

The focus of this study is the development of a proper process monitoring strategy for monitoring multiple correlated nonlinear profiles. This study examines two alternative solutions, in which profiles are first fitted by B-splines according to Chang and Yadama's method (2010). Then the deviations of the observed profile from the fitted profile are recorded to generate a vector of plotting statistics. A multivariate EWMA (MEWMA) control chart is then used for process monitoring. The first proposed method converts absolute deviations at each profile into a summary statistic. The second proposed method contains several numbers for each profile because a profile is segmented into $p$ sections where $p>1$, where $p$ is a constant to be determined from engineering knowledge or the complexity of a profile. Each section is represented by a summary absolute deviation statistic. A two-profile simulation study is conducted to characterize property of the proposed approaches in terms of ARL. We will also discuss the pros and cons of both approaches, and how we combine the salient features of both methods into a hybrid approach. The proposed hybrid method combines method I and method II, which monitors each section or segment by a MEWMA control chart. Multiple ( $p$ ) MEWMA charts need to be maintained for the proposed hybrid method. A real-world data set from a curing process is used to demonstrate the implementation of 
the proposed methods.

The organization of this study is the following. First, the modified Chang and Yadama's (2010) method is briefly summarized, as well as the MEWMA procedure, followed by the proposed methods. Second, the experimental design of the simulation is introduced for testing the robustness of the proposed methods. Third, the ARL property for all proposed methods is introduced. Finally, the discussion and conclusion will be drawn in this section.

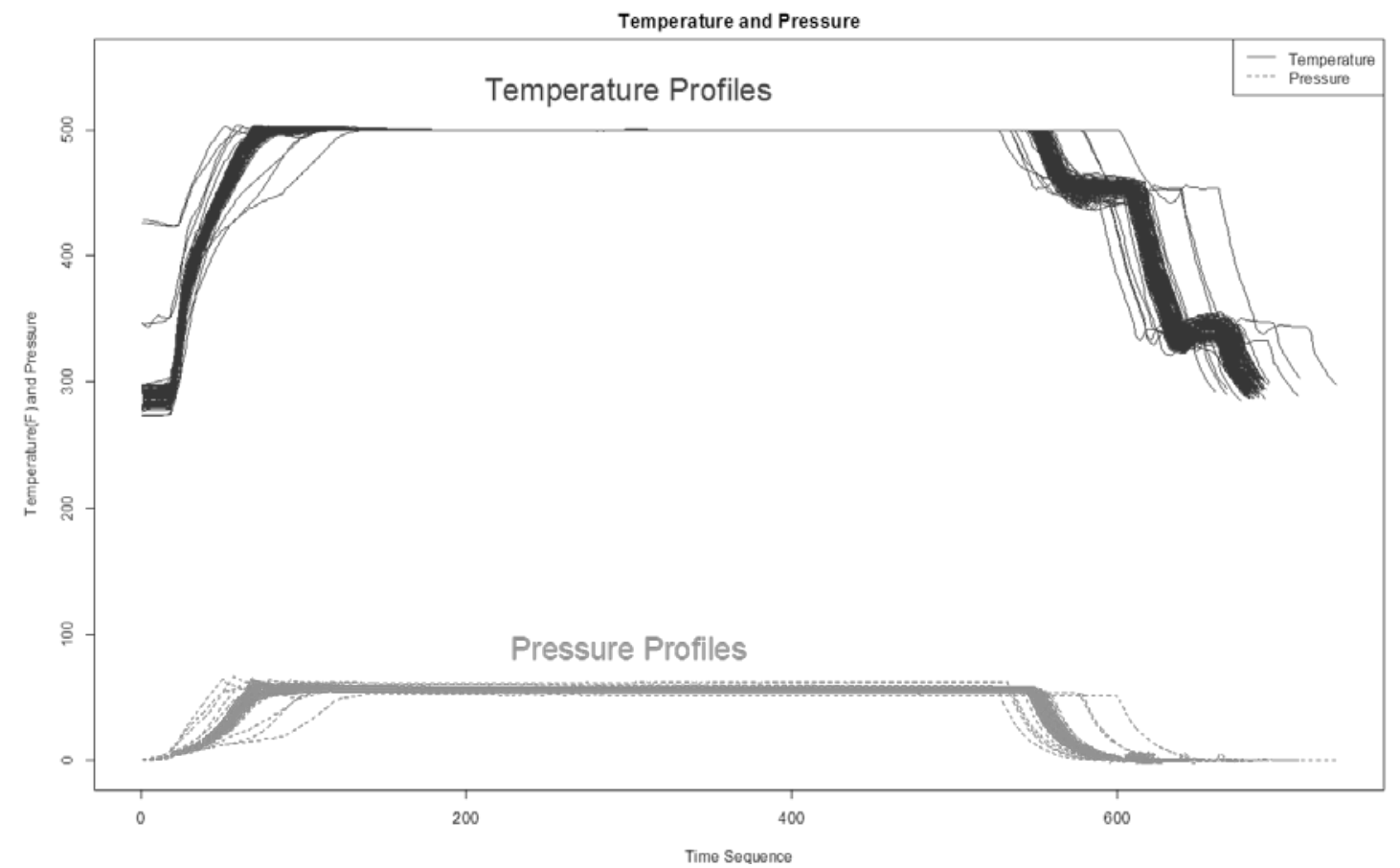

Figure 1 Overall air temperature and pressure profiles from the curing process of highpressure hose products.

\section{The Proposed Methods}

In this study, two methods, method I and method II, are proposed to monitor the stability of the process whose quality characteristics are multiple profiles. Both methods consist of one multivariate control chart for all profiles and share the common modelling treatment, i.e., the modified Chang and Yadama's method (see Chang and Yadama's, 2010). Therefore, this section summarizes the original Chang and Yadama's method followed by the modified version. Then, the MEWMA procedure is presented. Finally, the proposed method I and II are shown in the last part of this section.

\section{Modified Chang and Yadama's Method}

Chang and Yadama (2010) proposed a control charting framework to monitor nonlinear profiles in detecting shape changes. They proposed single segment and multiple segments approach for monitoring one profile. We will only introduce their multiple segments approach in this study because this approach provides more details of fault 
location for diagnosis purposes. The procedure of their proposed multiple segments approach is shown in Figure 2. Note that $D_{i j}$ in Step 5 can be presented as equation (1) where $\bar{x}_{j k}$ is the mean profile of segment $k$ that can be calculated by using B-spline fitting. Moreover, the MTY decomposition method shown in Step 7 can be found in Mason, Tracy, and Young's (2001) study for interpreting $T^{2}$ control chart signals.

$$
D_{i j}=\frac{\sum_{k=1}^{c}\left|x_{i j k}-\bar{x}_{j k}\right|}{c}, \quad i=1,2, \ldots, n ; j=1,2, \ldots, p,
$$

where $|\cdot|$ represents absolute value; $n$ is the number of profiles and $i$ is the index of profile; $p$ is the number of segments of a profile and $j$ is index of the segment; $c$ is the number of control points within each segment so that a B-spline can be fit to each segment, and $k$ is the index of control point. All numbers of $n, p$ and $c$ are known or well defined according to the manufacturing process and the complexity of a profile. The default $c$ is equal to 16 according to Chang and Yadama's study (2010).

Procedure of Chang and Yadama's Method
Step 1: Apply Discrete Wavelet Transformation (DWT) to the given profile to the
desired level, so that the DWT coefficients can be obtained.
Step 2: Reconstruct the DWT coefficients to original domain in two sets, signal of
mean and signal of variance.
Step 3: Partition the mean signal into $p$ segments.
Step 4: Apply B-spline with $c$ control points to each segment, so that a control point
matrix of order $n \times c \cdot p$ is constructed, where $n$ is the profile replicates.
Step 5: The mean distance difference vector $Y_{i}=\left[D_{i 1}, D_{i 2}, \ldots, D_{i p}\right]$ is calculated, in
which is associated with the control point matrix.
Step 6: To monitor the profile stability, the Hotelling's $T^{2}$ statistics on $Y_{i}$ are
calculated using the mean distance difference vectors.
Step 7: If process is in-control, go to Step 5; otherwise the MTY decomposition
method is used for identifying the responsible $T^{2}$ components.
Figure 2 Procedure of Chang and Yadama's Method.

For the curing example in this study, since the curing process is control by the PLC, the within profile variance is very small. In other words, noises in each profile are very small. Therefore, we do not consider the use of the discrete wavelet transformation (or DWT) method for modelling the variance profiles as originally proposed in steps 1 and 2 in Chang and Yadama (2010). Moreover, according to Chang and Chou's (2009) study, the B-spline fitting is sufficient without applying the DWT to the Chang and Yadama's method when the monitoring of the profile shape change is the only consideration. Therefore, the B-spline fitting technique is applied to construct the proposed control charts. In addition to these changes, a MEWMA control chart is selected to be the charting tool due to its sensitivity and flexibility. In addition, the MTY decomposition method can be replaced by Chang and Chou's (2010) marginal cumulative sum (or CUSUM) glyphs because of its benefits of visualization and capability of dealing with high dimensional dataset of up to 20. The procedure of modified Chang and Yadama's method is shown in Figure 3. The MEWMA control chart is introduced in the next section. 


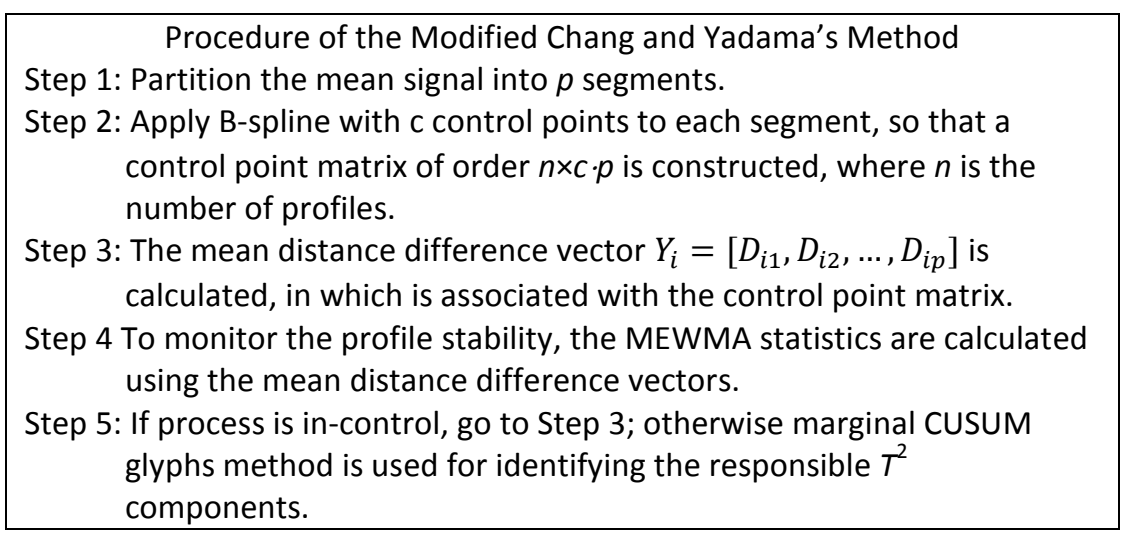

Figure 3 Procedure of Modified Chang and Yadama's Method

\section{Multivariate EWMA Control Chart}

The charting technique based on MEWMA is introduced in this section. The MEWMA was first developed by Lowry et al. (1992). It is the extension version of the EWMA for solving multivariate quality control problem. The procedure of MEWMA is presented as follows. The MEWMA statistics $T^{2}$ of the $i^{\text {th }}$ observation is shown in equation (2), where $Z_{i}$ is the extension form of univariate EWMA as shown in equation (3). Note that $0 \leq \lambda \leq 1$ and $Z_{0}=0$. The selection of chart parameters, $\lambda$ and $H$, can be found in Prabhu and Runger's (1997). The variance-covariance matrix of $Z$ 's, $\Sigma_{Z_{i}}$, can be calculated using equation (4), where $\Sigma$ is a variance-covariance matrix, which is either known or can be estimated from a phase I control charting procedure with $m$ individual observations according to equations (5), (6), and (7). Moreover, $\Sigma$, shown in the equation (5) performs better than that of using the conventional approach if there was no trend, cycle, etc., in the process. If the process was totally random, the variancecovariance structure determined by equation (5) and the conventional approach would have no difference (Holmes and Mergen, 1993).

$$
\begin{gathered}
T_{i}^{2}=Z_{i}^{\prime} \Sigma_{Z_{i}}^{-1} Z_{i} \\
Z_{i}=\lambda\left(x_{i}\right)+(1-\lambda) Z_{i-1} \\
\Sigma_{Z_{i}}=\frac{\lambda}{2-\lambda}\left[1-(1-\lambda)^{2 i}\right] \Sigma \\
\Sigma=\frac{V^{\prime} V}{2(m-1)} \\
V=\left[v_{1}^{\prime} v \cdots v_{m-1}^{\prime}\right]^{\prime} \\
v_{i}=x_{i+1}-x_{i}
\end{gathered}
$$

\section{Method I: One Chart for All Profiles and One Segment per Profile}

The first method proposed in this study is straightforward. We first apply the Step 1 to Step 5 of the modified Chang and Yadama's method in Figure 3 with one 
segment to each profile so that multiple profiles become a vector with each element representing one profile. As shown in equation (8), given a set of profiles, $X_{i}$ is the $i^{\text {th }}$ observation and $m$ is the number of types of profile and $D_{j}$ can be calculated by using equation (1) with the number of segment of 1 . For example, if the number of multiple profiles is two types with 512 data points in a profile, such as, temperature and pressure profiles of curing process of high-pressure hose products in this study, the number of types of profile $m$ is equal to two. Therefore, the $X_{i}$ is transformed from a matrix of size $2 \times 512$ into a $2 \times 1$ matrix. After Step 1 to Step 5 in Figure 3 is applied, the input variables expressed as equation (8) will be input into the MEWMA. Then a process will be stopped if any out-of-control signal takes place.

$$
X_{i}=\left[\begin{array}{llll}
D_{1} & D_{2} & \ldots & D_{j}
\end{array}\right]^{\prime},
$$

where $i$ is the index of observations and $j=1,2, \ldots, m$

\section{Method II: One Chart for All Profiles and Multiple Segments per Profile}

The second approach in this study is similar to the method I except that the number of segments of each type of profile $p$ is greater than 1 . The number of segments $p$ is defined by users' pre-knowledge about the process of interest. In our real world case, the curing process of high-pressure hose product, the $p$ is equal to three because the quality engineer in the $\mathrm{PH}$ cooperate specified the process consists of three stages. Users can also segment the profile based on the section of interests. The choice of $p$ is a balance of diagnostic need and computational resources. Once the number of the segments is determined by quality engineers, the procedure of the method II and method I are identical. The input variables are specified in equation (9), where $X_{i}$ represents the $i^{\text {th }}$ observation, $k$ is the index of segment, and $j$ is the index of profile. The advantage of using segmentation is that the quality engineers can gain more details of fault locations when a diagnosis is needed. In other words, the segmentation method is sensitive to partial profile shape changes. The main drawback of method II is that the type I error of the MEWMA will increase when the number of segments increases. For example, if the process consists of 5 profiles with 4 segments each, the vector $X_{i}$ will contain 20 elements while the one segment approach only has 5 elements in the vector.

$$
\begin{aligned}
& X_{i}=\left[D_{11}, D_{12}, \ldots, D_{k j}\right]_{i}^{\prime} \\
& \text { where } k=1,2, \ldots, p ; j=1,2, \ldots, m ; i \text { is the index of observations. }
\end{aligned}
$$

\section{A Simulation Study}

In order to study the performance of the proposed methods, all charts are established with in-control ARL approximately 200, denoted by $\mathrm{ARL}_{0}=200$. Moreover, the out-of-control ARL is denoted by $\mathrm{ARL}_{1}$ in the simulation study. $\mathrm{ARL}_{1}$ is used to evaluate the charting performance of the proposed approaches at the same false alarm rate. The simulation study conducts two correlated four-parameter logistic curves. The curve equation is adapted from Jensen and Birch (2009). They used this curve in their simulation study for generating ARL property and then applied this property to the real world case. Note that, their study only consider one type of profile. In this study, the 
curve equation will be extended to multiple profiles case. Specifically, in each sample, two correlated profiles are studied simultaneously. Equation (10) shows the operated function in this simulation study. Note that the coefficients in the equation (10), $A=5$, $B=8, C=0.6$, and $D=0$, are as same as the setup in Jensen and Birch's (2009) study. Further, to simulate the correlated multiple non-linear profiles, it is assumed that the parameters between profiles are generated from independently and identically multivariate normal distribution from one observation to another. If the parameters $a$ and $b$ are said to be generated from independently and identically multivariate normal distribution with mean vector $\mu$ and variance-covariance matrix $\Sigma$, it is denoted as $(a, b)^{\prime} \sim N M(\mu, \Sigma)^{\prime}$. The equation of simulated multiple correlated non-linear profiles is shown in equation (11) of which coefficients follow the following distribution: $\left(a_{0 i}, b_{0 i}\right)^{\prime}$ $\sim M N\left(\mu_{0}, \Sigma_{0}\right)^{\prime},\left(a_{1 i}, b_{1 i}\right)^{\prime} \sim M N\left(\mu_{1}, \Sigma_{1}\right)^{\prime}$, and $\left(e_{1 i}, e_{1 i}\right)^{\prime} \sim M N\left(\mu_{e}, \Sigma_{e}\right)^{\prime}$. Specific setup for the parameters of multivariate normal distribution is given in equation (12). Note that equation (10) determines the shape of two multiple correlated profiles in the simulation study. Following the profile property of the high-pressure hose products in the curing example, in which the noises within each profile are very small, and the variancecovariance matrix of error terms in equation (11) is denoted as $\Sigma_{e}$ specified by equation (12). An example of 25-pair in-control multiple correlated nonlinear profiles generated by equation (11) is shown in Figure 4.

$$
\begin{aligned}
& y_{i j}=A+\frac{D-A}{1+\left(\frac{x_{i j}}{C_{i}}\right)^{B_{i}}}+\epsilon_{i j} \text { for } i=1,2,3, \ldots, m ; j=1,2,3, \ldots, n_{i} \\
& \left\{\begin{array}{l}
f_{i j}=a_{0 i}+a_{1 i} y_{i j}+e_{1 i} \\
g_{i j}=b_{0 i}+b_{1 i} y_{i j}+e_{2 i}
\end{array}\right. \\
& \mu_{0}=(10,-5)^{\prime}, \mu_{1}=(10,3)^{\prime}, \Sigma_{0}=\left(\begin{array}{cc}
1 & \rho \\
\rho & 1
\end{array}\right), \Sigma_{1}= \\
& \left(\begin{array}{cc}
0.1^{2} & 0.01 \rho \\
0.01 \rho & 0.1^{2}
\end{array}\right), \text { and } \mu_{e}=(0,0)^{\prime}, \Sigma_{e}=\left(\begin{array}{cc}
0.1^{2} & 0.01 \rho \\
0.01 \rho & 0.1^{2}
\end{array}\right) \text { where }^{(12} \\
& \rho \text { is correlation parameter. }
\end{aligned}
$$

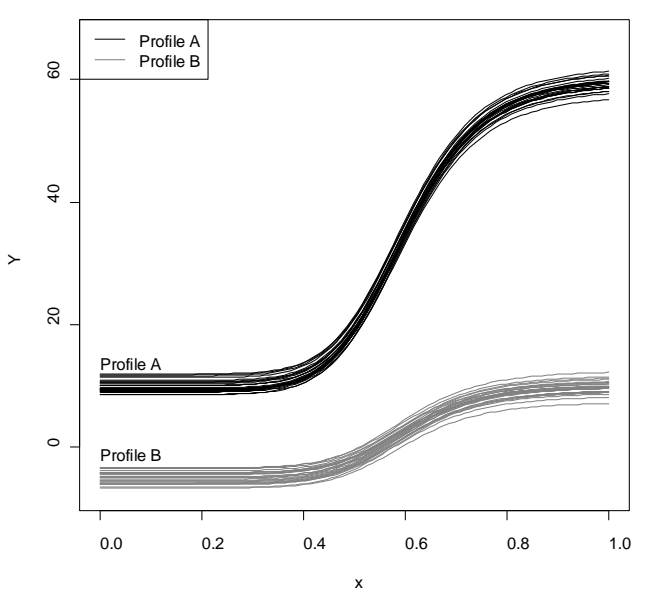

Figure 4 An example of 25-pair in-control profiles. 


\section{Experimental Design}

To test the robustness of the proposed methods, profiles shifted in shape is considered in this simulation study with various testing factors. Figure 5 shows the experimental design of the simulation study. In this research, only shape changes are considered due to the shape change could be caused by a number of factor combinations. Given two types of profiles, Profile A and Profile B, possible factor combinations include two categories and five scenarios for the profile shapes changes are given according the scheme in Figure 5 for the simulation study.

In addition to shifted types, correlations between profiles and shift sizes are also considered in this simulation study. For the factor of correlation between profiles, there are three attributes to be tested, i.e., low $(\rho=0.3)$, moderate $(\rho=0.5)$, and high correlated $(\rho=0.9)$. As for shift sizes, three magnitudes: small, medium, and large shift, are considered in this simulation study. The in-control ARL is fixed at approximately 200 for method I and method II. Therefore, there are total 45 cases to be tested for the proposed methods. Detailed parameter settings for the simulation study are discussed as follows:

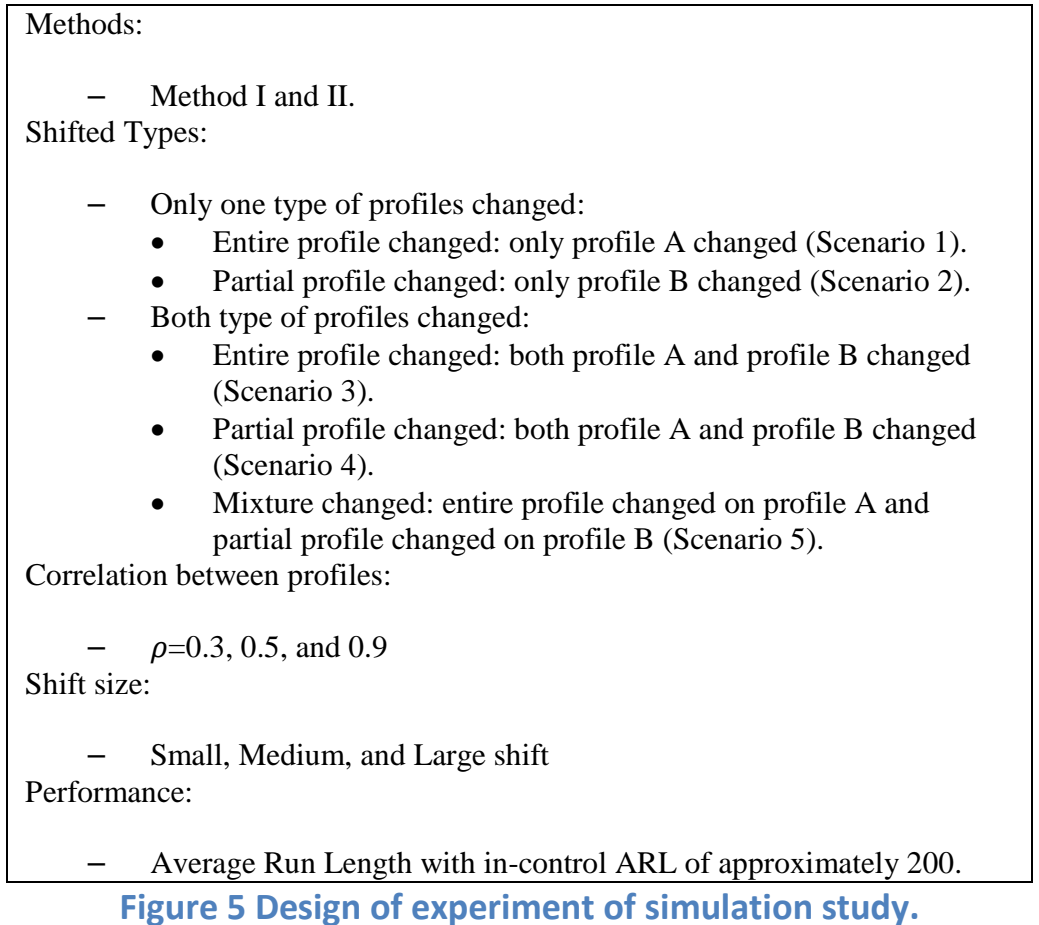

\section{Only the shape of either profile A or $B$ is shifted}

Scenario 1: The shape of profile A shifts entirely and the shape of profile $B$ unchanged

In this scenario, the shape of profile A is vertically shifted away from the reference profile. With respect to the real world case, the curing process for highpressure hose products, it is possible that the temperature profiles are shifted and go above the reference profile if the PLC or a thermocouple malfunctions, but the chamber is still airtight so that the pressure profiles are in control. This scenario may result in 
defective hose products due to overheating in the chamber. In this simulation study, we consider whether the shape of profile A is shifted entirely and go above the reference profile or not. Note that coefficient $a_{0}$ in equation (11) controls the vertical magnitudes change of profile A. Small shift is defined as the magnitude of $a_{0}$ from 10 to 12; medium shift is magnitude of $a_{0}$ from 10 to 14; and the large shift scenario is magnitude of $a_{0}$ from 10 to 16 . Figure 6 (a) shows the profiles in scenario 1 where the shape of profile A exhibits a large shift. Twenty-five in-control profiles and another 25 shifted profiles with correlation $\rho=0.5$ are superimposed on top of each other.

Scenario 2: The shape of profile B shifts partially and the shape of profile A unchanged

In this scenario, we alter $b_{1}$ in equation (11) to change the partial shape of profile B. Scenario 2 examines whether the proposed method is capable of detecting a leaking chamber during the operation process. As described in scenario 1, scenario 2 also considers three different correlations between profiles. Figure 6 (b) shows the graphics of scenario 2 profiles with large shift magnitudes along with $\rho=0.5$. From Figure 6 (b), profile $B$ changes at the middle of the profile. The magnitude of small shift in the simulation model is $b_{1}$ changed from 3 to 3.5. For medium shift, $b_{1}$ is changed from 3 to 4 , while $b_{1}$ changed from 3 to 4.5 represents large shift in this simulation study.

\section{Shapes of both profiles are shifted}

\section{Scenario 3: Shapes of both profiles A and B are shifted entirely}

This scenario simulates the case both shapes of profiles A and B are changed entirely. Since the parameters $a_{0}$ and $b_{0}$ control vertical shifts in equation (11), $a_{0}$ is altered to change the shape of profile A higher than the reference profile, and $b_{0}$ is manipulated to make the entire profile B shift below the reference profile. The setting of correlations for scenario 3 is the same as that in the scenario 1 and 2, but the magnitudes of shift sizes are different from the former settings and given as follows: (1) small shift: $a_{0}$ shifted from 10 to 12 and $b_{0}$ shifted from -5 to -3 ; (2) medium shift: $a_{0}$ shifted from 10 to 14 and $b_{0}$ shifted from -5 to -1 ; and (3) large shift: $a_{0}$ shifted from 10 to 16 and $b_{0}$ shifted from -5 to 1 . The superimposed profiles A and B with large shifted for scenario 3 is shown in Figure 6 (c). Scenario 3 intends to simulate the case where both temperature and pressure profiles are changed, especially when malfunctions take place in both thermocouples and pressure sensors or the door of vulcanizer is not sealed.

\section{Scenario 4: Shapes of both profiles A and B are partially changed}

Scenario 4 examines the case when shapes of both profiles are partially changed. In this case, profiles $A$ and $B$ are all in control before the middle point of the process, but both profile A and profile B are shifted after the middle point. Figure 6 (d) shows the overall profiles of A and B including shifted profiles with large shifted magnitude. 
The shift size in this scenario is as the follows: (1) small magnitude size of shift is simulated by altering $a_{1}$ from 10 to 10.5 and $b_{1}$ from 3 to 3.5; (2) medium shift is generated by changing $a_{1}$ from 10 to 11 and $b_{1}$ from 3 to 4 ; (3) large shift is created by changing the parameters $a_{1}$ and $b_{1}$ from 10 to 11.5 and 3 to 4.5 , respectively. The correlations setting for each profiles in this scenario is the same as scenario 1 as well.

Scenario 5: Shape of profile $A$ is shifted entirely and the shape of profile $B$ is shifted partially

The parameter settings for the profiles in scenario 5 are given as following: (1) small shift: $a_{0}$ shifted from 10 to 12 and $b_{1}$ shifted from 3 to 3.5; (2) medium shift: $a_{0}$ shifted from 10 to 14 and $b_{1}$ shifted from 3 to 4; (3) large shift: $a_{0}$ shifted from 10 to 16 and $b_{1}$ shifted from 3 to 4.5 . Specifically, we examine if the proposed method is capable of detecting changes when the shape of temperature profile $A$ is shifted entirely but the pressure profile $B$ is changed after half of the process. Figure 6 (e) shows the superimposed profile A and profile B with large shift magnitude.

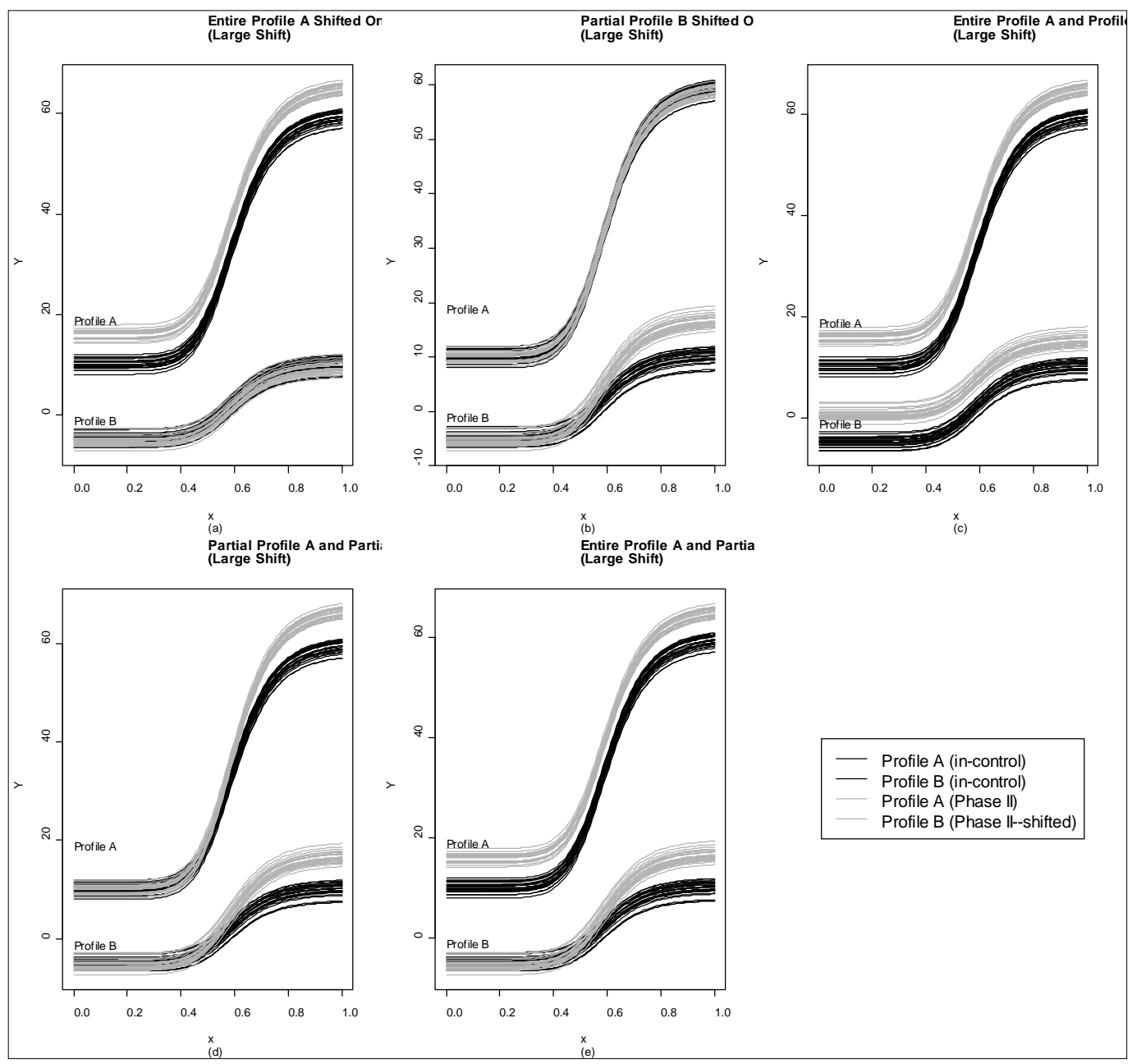

Figure 6 Scenarios of simulation study, (a) entire Profile A shifted only; (b) partial Profile B shifted only; (c) both Profile A and Profile B shifted entirely; (d) both Profile A and Profile B changed partially; (e) entire Profile A changed and partial Profile B changed. 


\section{Simulation Results and Discussion}

Simulations according to the scenarios 1 to 5 described in the previous section are conducted. The property of method I and method II is characterized by both incontrol and out-of-control ARL values. We fixed the in-control $A R L_{0}=200$ for both methods. The out-of-control ARL, denoted by $\mathrm{ARL}_{1}$, is the smaller the better. The smallest $A_{R L}$ of both methods for all scenarios are shown in Table 1, which can also be used for MEWMA design since $\lambda$ and $H$ are readily available. In Table 1 , the values of $\mathrm{ARL}_{1}$ of both methods are close to each other when the correlation coefficient, $\rho$, is low or moderate. However, once $\rho$ is high, the $\mathrm{ARL}_{1}$ is dramatically decreased in all scenarios. For example, the $\mathrm{ARL}_{1}$ for the case of small shift of scenario 2 is 36.349 when $\boldsymbol{\rho}=0.3$, but it decreases to 19.9006 when $\boldsymbol{\rho}=0.9$. This crucial result confirms the research hypothesis that highly correlated profiles enhance detection power. This result also justifies the merit of the use of multivariate control charts to monitor all profiles simultaneously rather than monitoring one profile at a time.

Table 1 The smallest ARL $L_{1}$ method I method II under different correlation structures in all scenarios.

\begin{tabular}{|c|c|c|c|c|c|c|c|}
\hline & & \multicolumn{2}{|c|}{$\rho=0.3$} & \multicolumn{2}{|c|}{$\rho=0.5$} & \multicolumn{2}{|c|}{$\rho=0.9$} \\
\hline \multirow[b]{2}{*}{ Scenario } & \multirow[b]{2}{*}{ Shift Size } & Method I & Method II & Method I & Method II & Method I & Method II \\
\hline & & $\begin{array}{c}\lambda=0.1 \\
H=14.03\end{array}$ & $\begin{array}{c}\lambda=0.1 \\
H=25.90\end{array}$ & $\begin{array}{c}\lambda=0.1 \\
H=14.49\end{array}$ & $\begin{array}{c}\lambda=0.1 \\
H=28.28\end{array}$ & $\begin{array}{c}\lambda=0.1 \\
H=14.49\end{array}$ & $\begin{array}{c}\lambda=0.1 \\
H=33.01\end{array}$ \\
\hline \multirow[t]{3}{*}{1} & Small & 2.981 & 3.994 & 2.922 & 4.140 & 1.695 & 2.103 \\
\hline & Medium & 1.480 & 1.827 & 1.468 & 1.873 & 1.205 & 1.259 \\
\hline & Large & 1.101 & 1.233 & 1.107 & 1.253 & 1.061 & 1.081 \\
\hline \multirow[t]{3}{*}{2} & Small & 36.349 & 2.477 & 37.816 & 2.562 & 19.901 & 1.706 \\
\hline & Medium & 18.203 & 1.480 & 18.407 & 1.555 & 9.160 & 1.069 \\
\hline & Large & 10.474 & 1.136 & 10.470 & 1.171 & 5.333 & 1.018 \\
\hline \multirow[t]{3}{*}{3} & Small & 1.753 & 2.369 & 1.753 & 2.604 & 1.513 & 1.914 \\
\hline & Medium & 1.070 & 1.195 & 1.055 & 1.244 & 1.006 & 1.260 \\
\hline & Large & 1.001 & 1.006 & 1.000 & 1.006 & 1.000 & 1.001 \\
\hline \multirow[t]{3}{*}{4} & Small & 18.390 & 1.385 & 20.123 & 1.552 & 25.526 & 2.181 \\
\hline & Medium & 9.389 & 1.054 & 10.341 & 1.131 & 13.240 & 1.471 \\
\hline & Large & 5.696 & 1.003 & 6.339 & 1.015 & 8.163 & 1.173 \\
\hline \multirow[t]{3}{*}{5} & Small & 2.907 & 1.730 & 2.968 & 1.791 & 2.048 & 1.151 \\
\hline & Medium & 1.476 & 1.101 & 1.497 & 1.120 & 1.208 & 1.039 \\
\hline & Large & 1.103 & 1.004 & 1.110 & 1.006 & 1.044 & 1.006 \\
\hline
\end{tabular}

Moreover, method I performs better (i.e. smaller ARL1 values) in scenario 1 and scenario 3 comparing to method II, while the method II has lower $\mathrm{ARL}_{1}$ in scenario 2, 4 and 5 than method I. In summary, when the shape of a profile change entirely, such as, scenario 1 and 3, method I is recommended. Otherwise, method II is recommended for the other cases, such as, scenarios 2, 4, and 5, where profiles shift partially. In practice, one may not know whether a profile may shift entirely or partially. It is up to the quality engineers to collect process data and identify the majority of the scenarios during a phase I study. Details of the proposed methods property, $A R L_{0}$ and $A R L_{1}$, for all five scenarios in the simulation study are shown in Table A1 and Table A2 in the Appendix.

We suggest the following strategy for users to implement the proposed methods. First, users should determine whether segmentations of profiles are appropriate or not. Second, they should also examine whether a linear model or nonlinear model can be 
used to fit the entire profile or a segment of a profile. For example, the shape of temperature profiles in the curing process consists of three stages the heat-up stage, the curing stage and the cool-down stage. If users need to know at what stage a profile may be out of control, then profiles need to be divided into three segments. A linear model is adequate for the curing stage since the profile shape in this stage is a straight line. However, nonlinear models are needed to fit the warm-up stage and the cool-down stage. If users are confident in their process is stable and they only intent to know whether the process is in-control or not, method I is capable of fulfilling this need with its simplicity. Nonlinear models should be used to fit the entire temperature and pressure profiles.

If the number of segments times the number of profile types is larger than 10, the conventional multivariate control chart may lose its effectiveness (Montgomery, 2009) such that the proposed methods work worse than the nominal performance. To deal with this issue, users can combine method I and method II together. In other words, users can apply the hybrid method to separate process monitoring into stages if the process stages are well defined. Each stage can be treated as one complete profile period and method I can be applied to each stage. For instance, if the process can be divided into three segments, users can construct three multivariate control charts for the process. Note that each multivariate control chart is associated to each stage. The advantage of this method is that users can monitor the process and diagnose a potential problem at the end of a stage instead of the end of a process. This hybrid method maintains the effectiveness of the multivariate control chart.

In summary, the charting frameworks of method I, method II, and the hybrid method are summarized here for any general multiple profile problem with $\mathrm{p}$ profiles and $\mathrm{m}$ segments. We assume that all profiles can be segmented at the same locations. For method I, one MEWMA chart is maintained with the plotting statistics of a $\mathrm{p} \times 1$ vector. Each element of this vector represents an average sum of deviations of a profile from its nominal profile. Method II also maintains one MEWMA chart but with the plotting statistics of a $\mathrm{mp} \times 1$ vector. Each element of this vector is the average sum of deviation of a segment instead of a profile. Finally, the hybrid method maintains m EWMA charts with the plotting statistics of a $\mathrm{p} \times 1$ vector. Each element of this vector is the average sum of deviation of a segment. All segments are from their respective profiles. Each MEWMA is used during a particular segment only.

\section{A Case Study: a Curing Process of High-Pressure Hose Products}

In this section, the proposed charting framework for monitoring multiple nonlinear correlated profiles is applied to a curing process that consists of temperature and pressure profiles for high-pressure hose products. PH Corporation seeking opportunities for improvement provides 154 air temperature and pressure profiles of phase I data. All 154 profiles are superimposed in Figure 1. Also, based on the PLC setting for temperature, each profile was divided into three segments, the heat-up stage, 
curing stage, and cool-down stage. Therefore, $p=3$ is given for method II in the process. Since two kinds of profiles are involved, $m=2$ in equation (9).

Those 154 profiles are prepared for phase I control charting. In Figure 1, it is easy to visually identify some out-of-control profiles due to their shapes are significantly different from the major group. Figure 7 shows all abnormal profiles, characterized by solid lines, as well as in-control profiles, shown in shadow. Note that the abnormal profiles are excluded from the phase I control charting. Figure 8 shows the MEWMA control charts on the cleaned data set using (a) method I and (b) method II. From these figures, we observe that all data points are in control with its associated control limit $H$. Therefore, the control limit $H$, mean vector, and variance-covariance matrix generated from method I and method II are used in phase II process for further process monitoring.

The issue of losing effectiveness using the multivariate control chart caused by the number of input variables larger than 10 may happen when the number of segments and number of profile types are large. To deal with this problem, the hybrid method is used to constructs three MEWMA charts for heat-up, curing, and cool-down stage, respectively. Figure 9 shows the phase I MEWMA control charts for these three stages using the hybrid methods. Note that, since the number of profile types in each control chart is two, users can use the same control limit $H$ as in method I, i.e., $H=14.03$. By using this hybrid method, users can not only diagnose which stage is responsible for the out-of-control signal, but also examine the stability at the end of the stage instead of at end of the entire process. For example, if the MEWMA control chart in the heat-up stage signal for out-of-control, operators can determine to stop the curing process or not, and start to diagnose potential causes at the end of that heat-up stage rather than at the end of the curing process. Early detection of process faults provides energy savings and assures product quality.

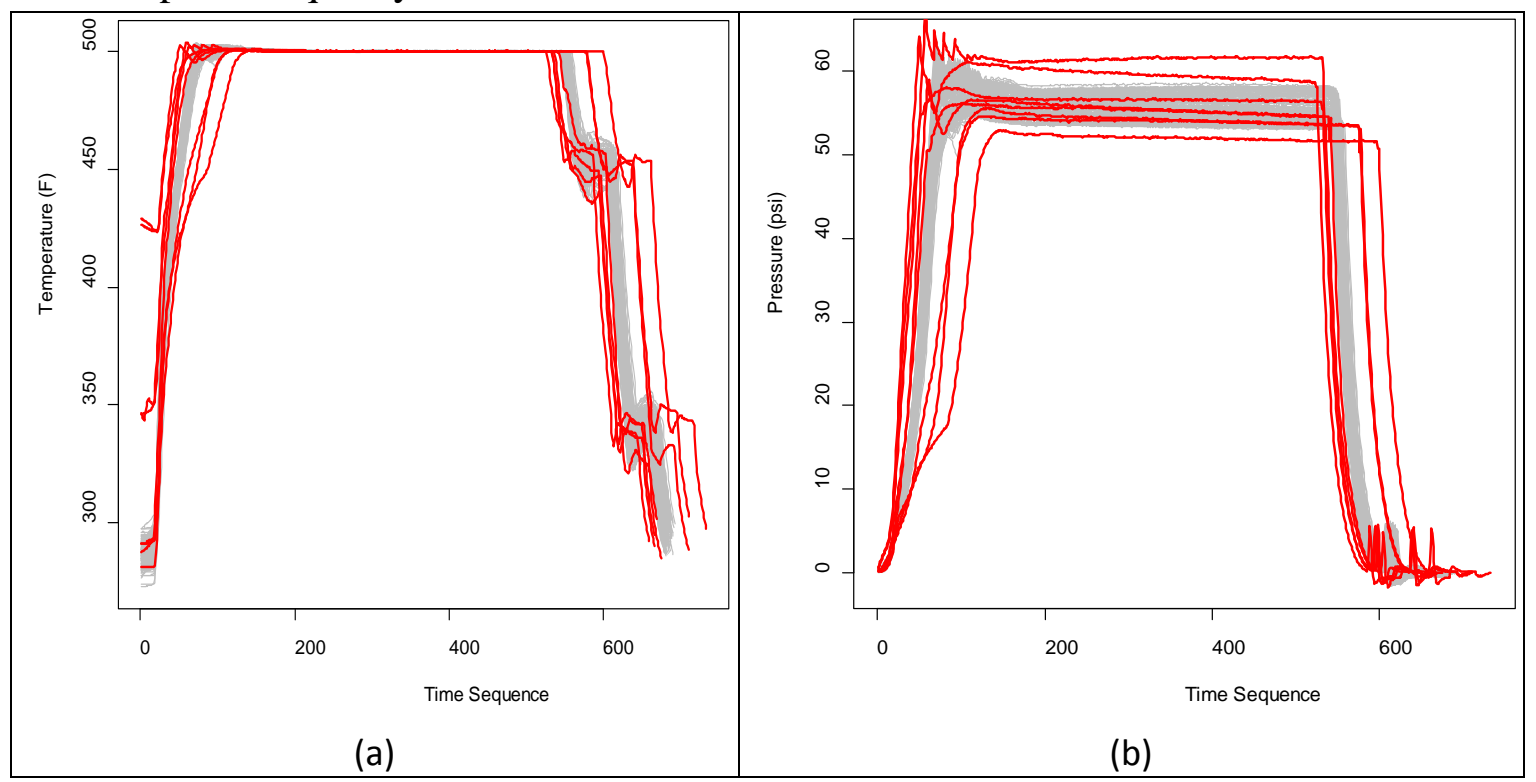

Figure 7 Abnormal (a) temperature profiles and (b) pressure profiles superimposed on incontrol profiles. 


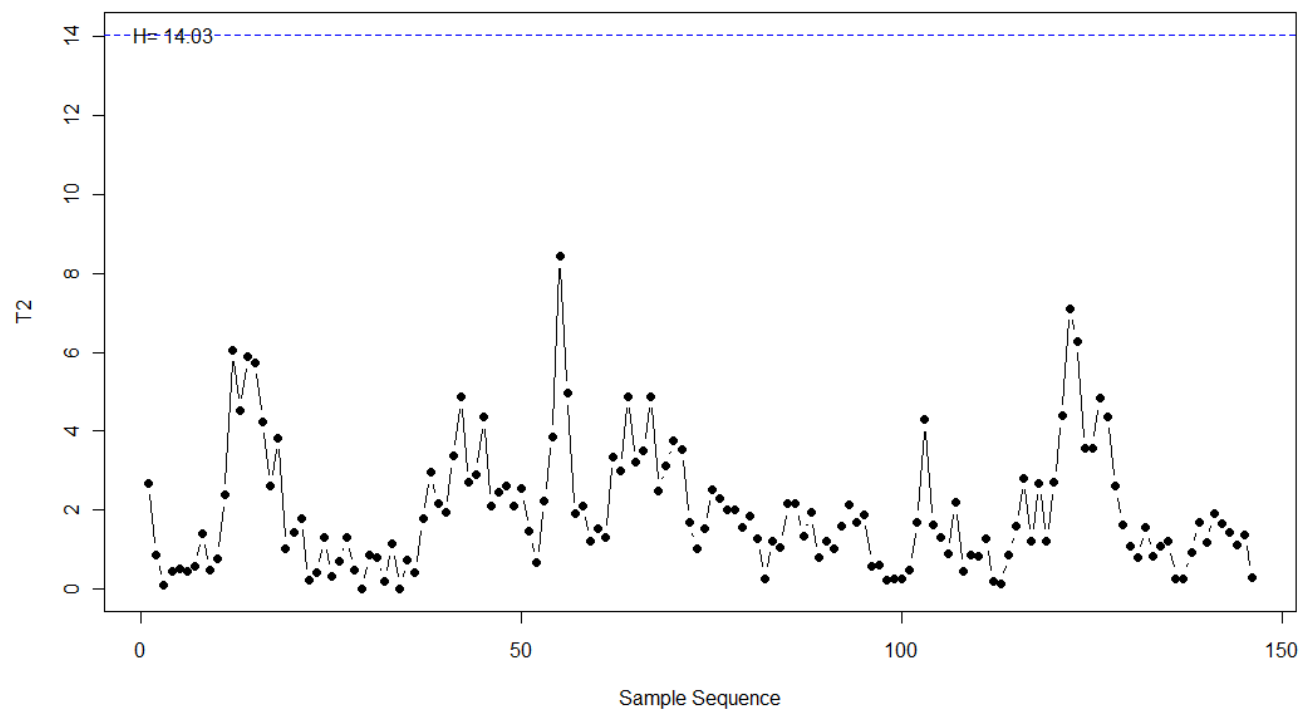

(a)

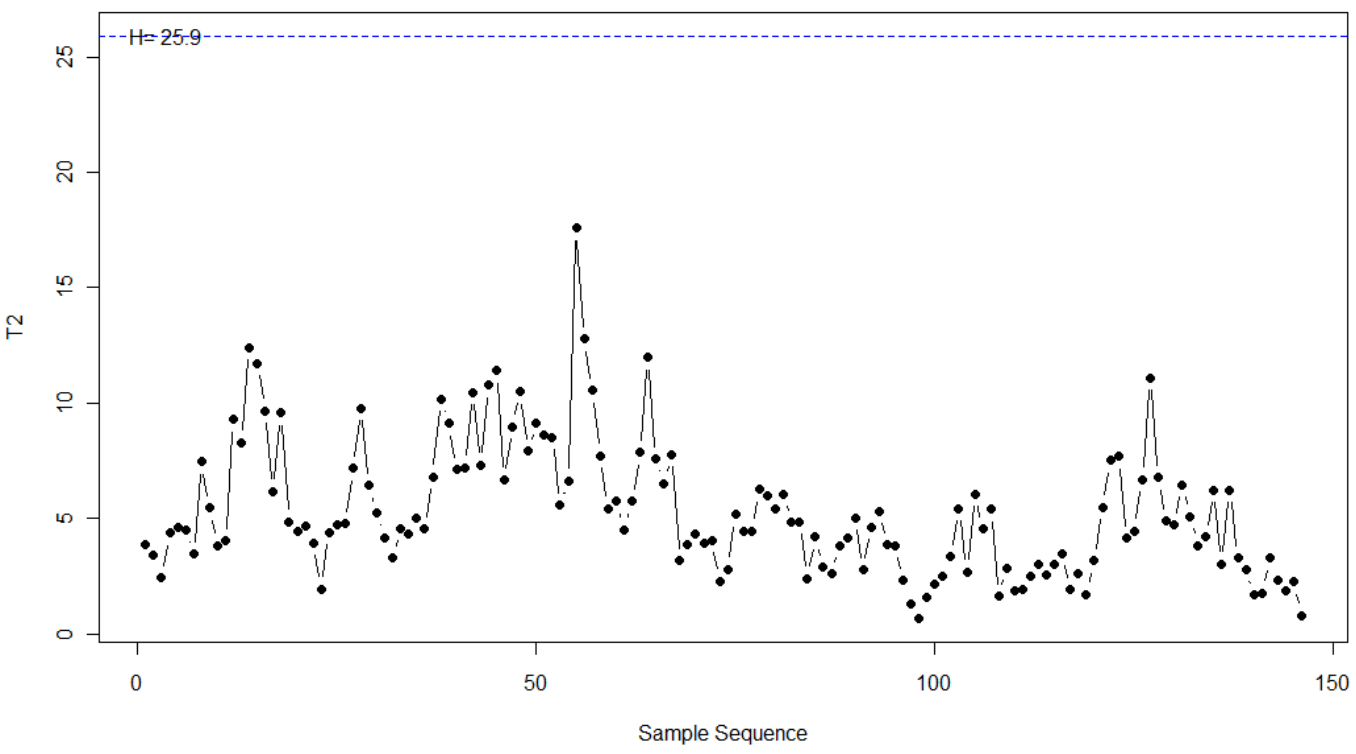

(b)

Figure 8 The phase I process MEWMA control charts of curing process of high-pressure hose products using (a) method I and (b) method II. 

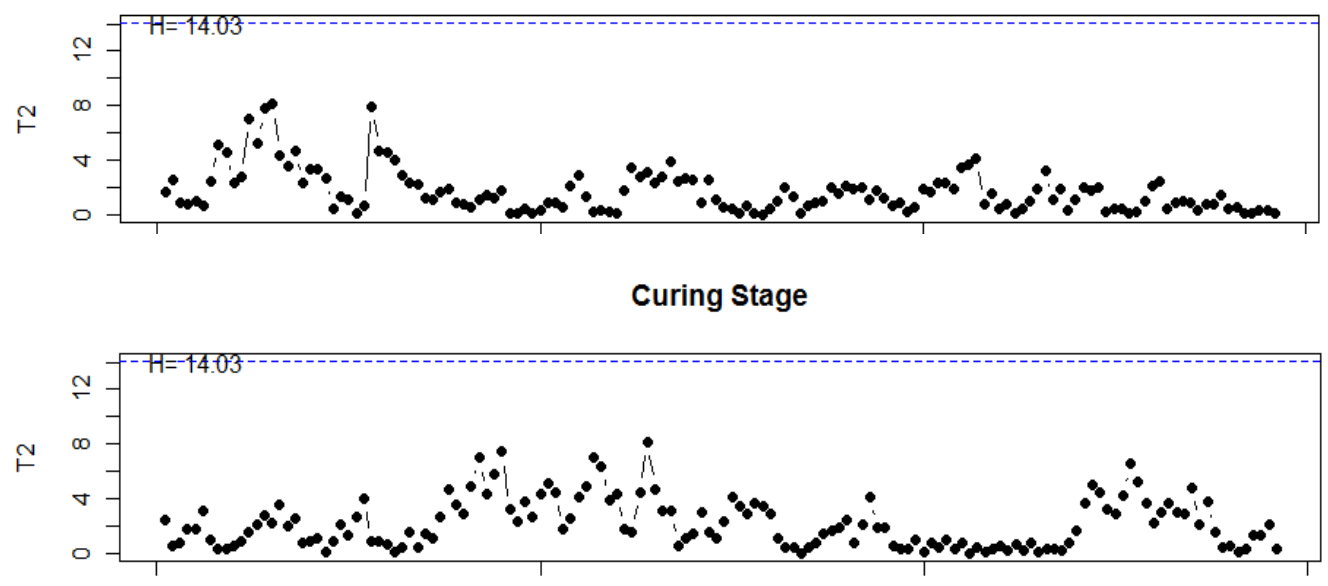

Cool-down Stage

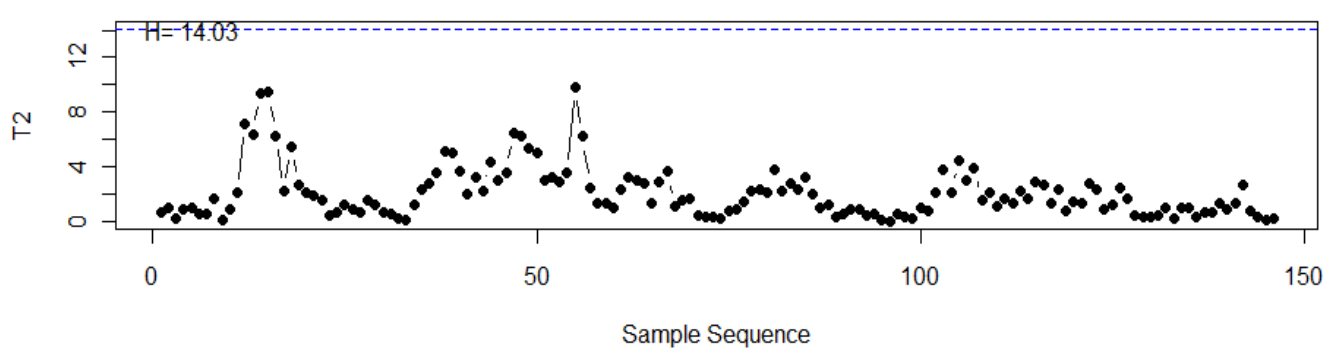

Figure 9 phase I control charts of the curing process using the hybrid method.

\section{Conclusion and Future Study}

Most profile analysis research up to date deals with the monitoring of single profile, but applications, such as, the hydraulic press machine and the curing process of high-pressure hose products, contain multiple correlated profiles for process monitoring. Although the monitoring of each type of profiles independently may be considered as an approach for detecting abnormal ones, high false alarm and low detecting power may be a consequence when given profiles are highly correlated. This study aims to tackle this problem by providing a framework for the process monitoring when the process consists of multiple linear or nonlinear correlated profiles. The proposed method I applies the modified Chang and Yadama's method that extends one profile process monitoring method (with $m$ segments) to a multiple profile solution (each profile representing a segment). The proposed method II divides each profile into $p$ segments first, and then applies the modified Chang and Yadama's method to each segment. Therefore, the multiple-profile problem can be considered as a multivariate statistical process control problem with the number of input variables $m \times p$. According to the simulation study, the method I has better performance in terms of out-of-control ARL 
values when the shape of profiles is changed entirely in the process, and method II is more sensitive when the process experiences partial profiles changes.

Additionally, the simulation results show that when profiles are highly correlated, the detecting power of the proposed method is much better than those cases with lower correlations. In a real-world case study, both method I and method II are capable of constructing the phase I process control chart if the product of the number of segments and the number of profile types is smaller than 10. The hybrid method that combines method I and method II are also investigated to provide quality engineers a broader view of diagnosis and maintains the effectiveness of detecting power. Moreover, since the proposed methods are sharing the common treatment of Chang and Yadama's (2010) method, they can handle the different shapes from other applications as long as profiles in the process have their own desired shapes with gold reference profiles existed. For example, the shape of temperature and pressure profiles generated from a curing process of high-pressure hose products follow their respective shapes, and the proposed methods all construct the phase I control chart without generating any false alarm signals.

Although the proposed methods show capability of dealing with multiple nonlinear profiles on monitoring products' quality perspective, they have some limitations. First, although the method I is capable of detecting entire shape shifted scenario, it lack of diagnosis ability when the process goes out-of-control. Second, even though the method II provides quality engineers more information in regards to diagnostic purpose, it will be less efficient if the number of segments times the profile types is over 10. Finally, the hybrid method takes care of those disadvantages that provided by the method I and method II, but it will be distract if there are too many segments have been defined. The future study of profile analysis should be extended to cover all the disadvantages of the proposed methods and to be applied for more complicated data sets, such as, the data sets of images and spatial surface. 


\section{Reference}

Chang, S.I. and Chou, S.H., 2009. A Study of Using Wavelet Transformation and BSpline Approximation for Nonlinear Profiles Monitoring, Proceedings of the 2009 Industrial Engineering Research Conference (IERC), 1567-1572, Miami, Florida, May 30-June 03.

Chang, S.I. and Chou, S.H., 2010. A Visualization Decision Support Tool for Multivariate SPC Diagnosis Using Marginal CUSUM Glyphs, Quality Engineering, 22(3), 182-198.

Chang, T. C., Gan, F. F., 2006. Monitoring Linearity of Measurement Gauges. Journal of Statistical Computation and Simulation, 76(10), 889-911.

Chang, S.I., Tsai, T.R., Lin, D.K.J., Chou, S.H., and Lin, Y.S., 2012. Statistical Process Control for Monitoring Nonlinear Profiles: A Six Sigma Project on Curing Process, Quality Engineering, 24(2), 251-263.

Chang, S.I. and Yadama, S., 2010. Statistical Process control for Monitoring Non-linear Profiles using Wavelet Filtering and B-Spline Approximation, International Journal of Production Research, 48(4), 1049-1068.

Chicken, E. Pignatiello, Jr., J., andSimpson, J.R., 2009. Statistical Process Monitoring of Nonlinear Profiles Using Wavelets, Journal of Quality Technology, 41(2), 198-212.

Chen, S. and Nembhard, H.B., 2011. A High-dimensional Control Chart for Profile Monitoring, Quality and Reliability Engineering International, 27(4), 451-464.

Ding, Y., Zeng, L., and Zhou, S., 2006. Phase I Analysis for Monitoring Nonlinear Profiles in Manufacturing Processes, Journal of Quality Technology, 38(3), 199216.

Holmes, D. S. and Mergen, A. E., 1993. Improving the Performance of the T2 Control Chart, Quality Engineering, 5(4), 619-625.

Jensen, W.A. and Birch, J.B., 2009. Profile Monitoring Via Nonlinear Mixed Models, Journal of Quality Technology 41(1), 18-34.

Jensen, W.A., Birch, J.B., and Woodall, W.H., 2008. Monitoring Correlation within Linear Profiles using Mixed Models, Journal of Quality Technology, 40(2), 167183.

Jin, J., and Shi, J., 1999. Feature-Preserving Data Compression of Stamping Tonnage Information Using Wavelets, Technometrics 41(4), 327-339.

Kazemzadeh, R.B., Noorossana, R., Amiri, A., 2008. Phase I Monitoring of Polynomial Profiles, Communications in Statistics-Theory and Methods, 37(10), 16711686.

Kang, L.; Albin, S. L., 2000. On-Line Monitoring when the Process Yields a Linear Profile, Journal of Quality Technology, 32(4), 418-426.

Kim, K., Mahmoud, M. A. and Woodall, W. H., 2003. On the Monitoring of Linear Profiles, Journal of Quality Technology, 35, 317-328.

Lowry, C.A., Woodall, W.H., Champ, C.W., and Rigdon, S.E., 1992. A Multivariate Exponentially Weighted Moving Average Control Chart, Technometrics, 34(1), 46-53. 
Mahmoud, M. A., 2008. Phase I Analysis of Multiple Regression Linear Profiles, Communications in Statistics - Simulation and Computation, 37(10), 2106-2130.

Mason, R.L., Tracy, N.D., and Young, J.C., 2001. A Practical Approach for Interpreting Multivariate T2 Control Chart Signals, Journal of Quality Technology, 29 (4), $396-406$.

Montgomery, D. C., 2009. Introduction to statistical quality control. New York, NY: John Wiley \& Sons.

Noorossana, R., Amiri, A., and Soleimani, P., 2008. On the Monitoring of Autocorrelated Linear profiles, Communications in Statistics-Theory and Methods, 37(3), 425-442.

Noorossana, R, Eyvazian, M., Vaghefi, A., 2010. Phase II Monitoring of Multivariate Simple Linear Profiles, Computers \& Industrial Engineering, 58(4), 563-570.

Noorossana, R., Saghaei, A., and Amiri, A., 2011. Statistical Analysis of Profile Monitoring, Hoboken, New Jersey: John Wiley \& Sons, Inc.

Paynabar, K. and Jin, J., 2011. Characterization of Non-linear Profiles Variations using Mixed-effect Models and Wavelets, IIE Transactions, 43(4), 275-290.

Prabhu, S.S. and Runger, G.C., 1997. Designing a Multivariate EWMA Control Chart, Journal of Quality Technology, 29(1), 8-15.

Qiu, P., Zou, C. and Wang, Z., 2010. Nonparametric Profile Monitoring by Mixed Effects Modeling, Technometrics, Vol. 52(3):265-277.

Reis, M.S., SARAIVA, P.M., 2006. Multiscale Statistical Process Control of Paper Surface Profiles, Quality Technology and Quantitative Management, 3(3), 263282.

Shiau, J.J.H., Huang, H.L., Lin, S.H., and Tsai, M.Y., 2009. Monitoring Nonlinear Profiles with Random Effects by Nonparametric Regression, Communications in Statistics-Theory and Methods, 38(10), 1664-1679.

Walker, E. and Wright, S. P., 2002. Comparing Curves Using Additive Models, Journal of Quality Technology, 34(1), 118-129.

Williams, J. D., Woodall, W. H., and Birch, J. B., 2007. Statistical Monitoring of Nonlinear Product and Process Quality Profiles, Quality \& Reliability Engineering International, 23(7), 925-941.

Woodall, W.H., 2007. Current Research on Profile monitoring, Produção 17(3), 420425.

Zou, C.,Tsung, F., and Wang, Z., 2007. Monitoring General Linear Profiles Using Multivariate Exponentially Weighted Moving Average Schemes, Technometrics, 49(4), 395-408. 


\section{Appendix}

TableA 1 The average run length of method I with three different correlation structures.

\begin{tabular}{|c|c|c|c|c|c|c|c|c|c|}
\hline \multicolumn{10}{|c|}{ Method I } \\
\hline \multicolumn{10}{|c|}{$\rho=0.3$} \\
\hline$\lambda$ & 0.1 & 0.2 & 0.3 & 0.4 & 0.5 & 0.6 & 0.7 & 0.8 & 0.9 \\
\hline $\mathrm{H}$ & 14.03 & 15.81 & 16.97 & 17.86 & 18.61 & 19.13 & 19.55 & 19.87 & 19.98 \\
\hline $\mathrm{ARL}_{0}$ & 200.170 & 199.925 & 199.809 & 200.167 & 199.617 & 199.829 & 199.840 & 200.167 & 199.809 \\
\hline Scen. 1 (small) & 2.981 & 3.203 & 3.400 & 3.614 & 3.896 & 4.242 & 4.705 & 5.275 & 5.987 \\
\hline Scen. 1 (medium) & 1.480 & 1.554 & 1.608 & 1.665 & 1.717 & 1.760 & 1.821 & 1.897 & 2.014 \\
\hline Scen. 1 (large) & 1.101 & 1.125 & 1.142 & 1.162 & 1.174 & 1.184 & 1.195 & 1.205 & 1.214 \\
\hline Scen. 2 (small) & 36.349 & 49.035 & 59.258 & 68.725 & 76.818 & 83.557 & 88.074 & 94.022 & 99.801 \\
\hline Scen. 2 (medium) & 18.203 & 24.604 & 30.988 & 37.398 & 43.991 & 49.290 & 55.851 & 62.033 & 66.700 \\
\hline Scen. 2 (large) & 10.474 & 13.208 & 16.867 & 21.015 & 23.316 & 29.945 & 34.551 & 39.603 & 44.195 \\
\hline Scen. 3 (small) & 1.753 & 1.886 & 1.976 & 2.053 & 2.163 & 2.277 & 2.432 & 2.662 & 2.886 \\
\hline Scen. 3 (medium) & 1.070 & 1.091 & 1.107 & 1.122 & 1.135 & 1.144 & 1.155 & 1.164 & 1.173 \\
\hline Scen. 3 (large) & 1.001 & 1.001 & 1.002 & 1.002 & 1.003 & 1.004 & 1.004 & 1.004 & 1.004 \\
\hline Scen. 4 (samll) & 18.390 & 23.135 & 27.833 & 32.099 & 36.519 & 40.005 & 43.883 & 47.784 & 51.197 \\
\hline Scen. 4 (medium) & 9.389 & 11.178 & 13.228 & 15.711 & 18.580 & 21.055 & 23.962 & 27.048 & 29.549 \\
\hline Scen. 4 (large) & 5.696 & 6.504 & 7.522 & 8.668 & 10.082 & 11.653 & 13.457 & 15.642 & 18.002 \\
\hline Scen. 5 (small) & 2.907 & 3.135 & 3.301 & 3.493 & 3.743 & 4.047 & 4.386 & 4.935 & 5.499 \\
\hline Scen. 5 (medium) & 1.476 & 1.545 & 1.594 & 1.641 & 1.696 & 1.732 & 1.785 & 1.864 & 1.953 \\
\hline Scen. 5 (large) & 1.103 & 1.128 & 1.145 & 1.163 & 1.175 & 1.186 & 1.194 & 1.205 & 1.217 \\
\hline \multicolumn{10}{|c|}{$\rho=0.5$} \\
\hline$\lambda$ & 0.1 & 0.2 & 0.3 & 0.4 & 0.5 & 0.6 & 0.7 & 0.8 & 0.9 \\
\hline $\mathrm{H}$ & 14.49 & 16.26 & 17.55 & 18.52 & 19.28 & 19.83 & 20.3 & 20.57 & 20.72 \\
\hline$A R L_{0}$ & 199.870 & 199.829 & 200.106 & 200.249 & 199.916 & 199.809 & 200.152 & 200.166 & 199.870 \\
\hline Scen. 1 (small) & 2.922 & 3.163 & 3.369 & 3.571 & 3.834 & 4.165 & 4.638 & 5.218 & 5.852 \\
\hline Scen. 1 (medium) & 1.468 & 1.541 & 1.592 & 1.646 & 1.701 & 1.748 & 1.811 & 1.886 & 1.983 \\
\hline Scen. 1 (large) & 1.107 & 1.132 & 1.155 & 1.172 & 1.185 & 1.198 & 1.210 & 1.221 & 1.238 \\
\hline Scen. 2 (small) & 37.816 & 49.977 & 61.750 & 71.478 & 80.470 & 86.518 & 92.114 & 96.633 & 102.777 \\
\hline Scen. 2 (medium) & 18.407 & 24.791 & 32.102 & 39.083 & 45.808 & 51.963 & 58.493 & 64.189 & 69.712 \\
\hline Scen. 2 (large) & 10.470 & 13.175 & 17.043 & 21.470 & 26.123 & 31.187 & 36.361 & 41.304 & 46.366 \\
\hline Scen. 3 (small) & 1.753 & 1.891 & 2.002 & 2.115 & 2.255 & 2.419 & 2.654 & 2.930 & 3.265 \\
\hline Scen. 3 (medium) & 1.055 & 1.079 & 1.096 & 1.111 & 1.125 & 1.134 & 1.148 & 1.160 & 1.171 \\
\hline Scen. 3 (large) & 1.000 & 1.000 & 1.001 & 1.001 & 1.001 & 1.001 & 1.001 & 1.001 & 1.001 \\
\hline Scen. 4 (samll) & 20.123 & 25.168 & 30.797 & 35.059 & 40.091 & 43.785 & 47.510 & 51.021 & 55.109 \\
\hline Scen. 4 (medium) & 10.341 & 12.377 & 14.897 & 17.851 & 20.570 & 23.577 & 26.695 & 29.542 & 32.576 \\
\hline Scen. 4 (large) & 6.339 & 7.271 & 8.485 & 9.909 & 11.521 & 13.280 & 15.450 & 17.942 & 20.349 \\
\hline Scen. 5 (small) & 2.968 & 3.192 & 3.398 & 3.596 & 3.857 & 4.185 & 4.604 & 5.130 & 5.797 \\
\hline Scen. 5 (medium) & 1.497 & 1.569 & 1.623 & 1.677 & 1.727 & 1.770 & 1.837 & 1.913 & 2.026 \\
\hline Scen. 5 (large) & 1.110 & 1.135 & 1.154 & 1.174 & 1.186 & 1.194 & 1.210 & 1.220 & 1.236 \\
\hline \multicolumn{10}{|c|}{$\rho=0.9$} \\
\hline lambda & 0.1 & 0.2 & 0.3 & 0.4 & 0.5 & 0.6 & 0.7 & 0.8 & 0.9 \\
\hline $\mathrm{H}$ & 14.49 & 15.95 & 16.99 & 17.81 & 18.4 & 18.9 & 19.16 & 19.38 & 19.43 \\
\hline $\mathrm{ARL}_{0}$ & 199.995 & 199.809 & 199.743 & 199.731 & 199.840 & 200.167 & 200.167 & 200.152 & 200.098 \\
\hline Scen. 1 (small) & 1.695 & 1.741 & $\mid 1.767$ & 1.792 & 1.814 & 1.825 & 1.843 & 1.869 & 1.881 \\
\hline Scen. 1 (medium) & 1.205 & 1.219 & 1.229 & 1.236 & 1.244 & 1.250 & 1.253 & 1.259 & 1.261 \\
\hline Scen. 1 (large) & 1.061 & 1.067 & 1.073 & 1.076 & 1.078 & 1.082 & 1.084 & 1.085 & 1.085 \\
\hline Scen. 2 (small) & 19.901 & 24.360 & 29.931 & 35.278 & 41.769 & 48.012 & 53.213 & 58.377 & 62.349 \\
\hline Scen. 2 (medium) & 9.160 & 10.493 & 12.289 & 14.643 & 17.444 & 20.958 & 24.490 & 28.552 & 32.204 \\
\hline Scen. 2 (large) & 5.333 & 5.852 & 6.479 & 7.379 & 8.526 & 10.127 & 11.763 & 13.906 & 16.345 \\
\hline Scen. 3 (small) & 1.513 & 1.590 & 1.652 & 1.713 & 1.762 & 1.827 & 1.888 & 1.941 & 1.972 \\
\hline Scen. 3 (medium) & 1.006 & 1.020 & 1.038 & 1.049 & 1.062 & 1.073 & 1.081 & 1.088 & 1.091 \\
\hline Scen. 3 (large) & 1.000 & 1.000 & 1.000 & 1.000 & 1.000 & 1.000 & 1.000 & 1.000 & 1.000 \\
\hline Scen. 4 (samll) & 25.526 & 31.831 & 37.762 & 43.219 & 48.015 & 52.195 & 55.907 & 59.355 & 62.502 \\
\hline Scen. 4 (medium) & 13.240 & 16.166 & 19.269 & 22.902 & 26.093 & 29.443 & 32.566 & 35.929 & 39.076 \\
\hline Scen. 4 (large) & 8.163 & 9.380 & 10.997 & 12.814 & 14.774 & 17.386 & 19.817 & 22.445 & 25.021 \\
\hline Scen. 5 (small) & 2.048 & 2.136 & 2.196 & 2.258 & 2.326 & 2.405 & 2.481 & 2.603 & 2.747 \\
\hline Scen. 5 (medium) & 1.208 & 1.233 & 1.246 & 1.258 & 1.269 & 1.275 & 1.283 & 1.294 & 1.300 \\
\hline Scen. 5 (large) & 1.044 & 1.051 & 1.054 & 1.058 & 1.060 & 1.062 & 1.062 & 1.064 & 1.064 \\
\hline
\end{tabular}


TableA 2 The average run length of method II with three different correlation structures.

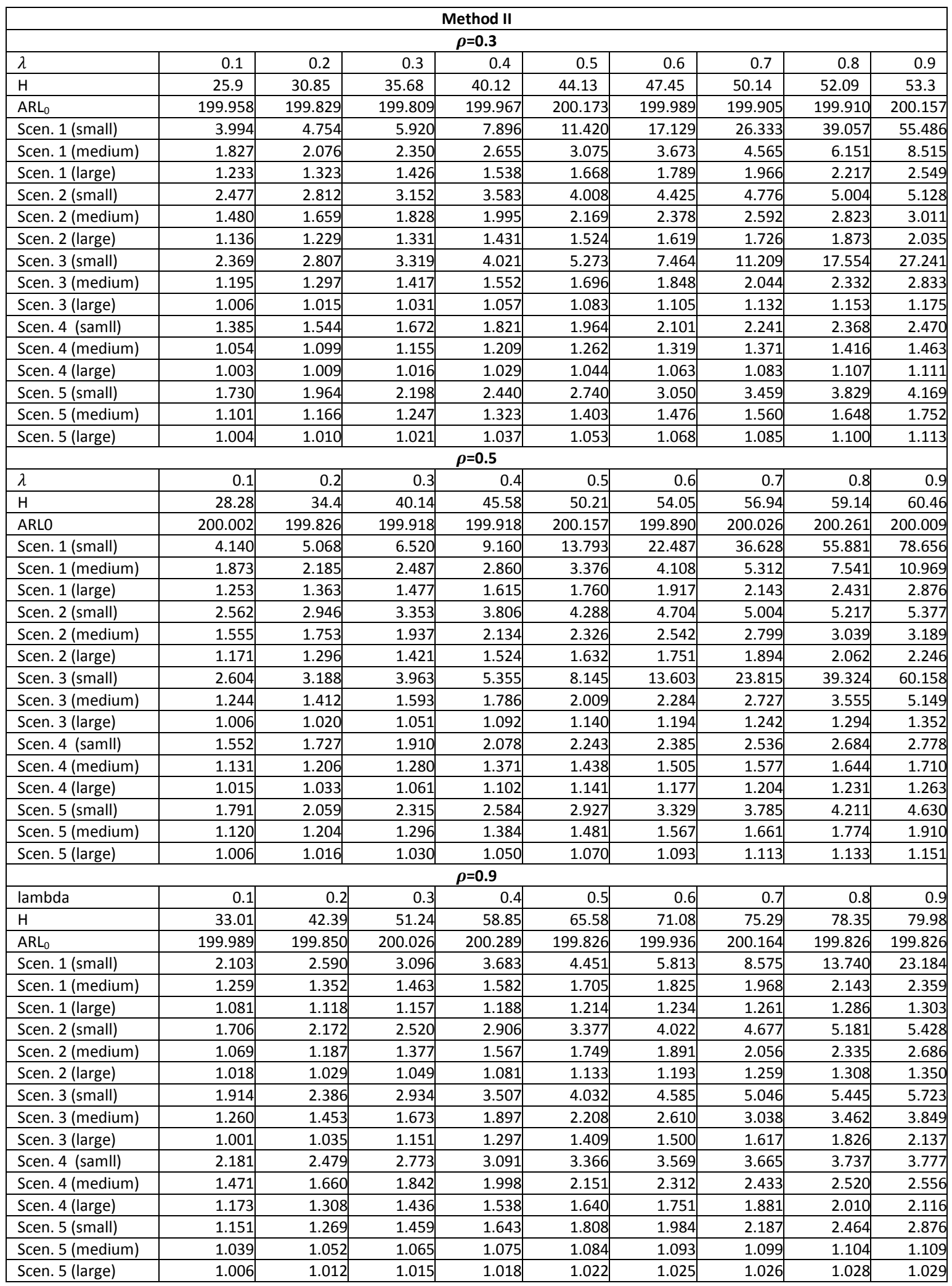

